\title{
USO DE REGULADORES DE CRESCIMENTO EM DOIS ESTÁDIOS, NO CONTROLE DO DOSSEL NA CULTURA DA SOJA RR, NO SUDOESTE GOIANO
}

\author{
ALMEIDA JÚNIOR, Joaquim Júlio ${ }^{1}$ \\ SOUSA, José Augusto Pereira ${ }^{2}$ \\ SMILJANIC, Katya Bonfim Ataides ${ }^{3}$ \\ RIBEIRO, Diego Oliveira ${ }^{4}$ \\ PEREIRA, Rogério Machado ${ }^{5}$ \\ PEROZINI, Alexandre Caetano ${ }^{6}$ \\ MIRANDA, Beatriz Campos ${ }^{7}$
}

\begin{abstract}
RESUMO: Cultivares modernas de soja com elevado potencial produtivo são amplamente testadas e difundidas na região, porém, o crescimento excessivo, podem causar perda no seu potencial de produtivo. Uma forma de resolver seria o uso de reguladores de crescimento para controlar o dossel. Este trabalho objetivou avaliar a biometria e a produtividade na cultura da soja, cultivar CD 2737 RR ao longo do desenvolvimento da cultura, após a aplicação de dois reguladores de crescimento em dois estádios de crescimento. $\mathrm{O}$ experimento foi conduzido por duas safras nos anos agrícolas de 2016/2017 e 2017/2018 na área experimental do Núcleo de Ensino e Pesquisa em Fitotecnia, apresentando como coordenadas geográficas, $17^{\circ} 58^{\prime} \mathrm{S}$ de latitude e $45^{\circ} 22^{\prime} \mathrm{W}$ de longitude e com $845 \mathrm{~m}$ de altitude, em um Neossolo Quartzarênico. O delineamento experimental foi em blocos casualizados em esquema 4x2x2+1 com quatro repetições. Os tratamentos foram com o herbicida Lactofem $240 \mathrm{gL}-1$, o regulador de crescimento Etefon 720 gL-1 em quatro doses de cada e dois estádios fenológicos e um controle sem aplicação. Foram coletados os dados das variáveis biométricas como: produtividade em sacas por hectare, peso de mil grãos, número de vagens por planta, número de grãos por planta, número de grãos por vagens, número de nós por planta, altura de planta e altura de inserção da primeira vagem na planta. Os dados foram analisados pelo programa Sisvar e submetidos à análise de variância, sendo as médias comparadas pelo teste t. Pode-se concluir que os reguladores de crescimentos utilizados em dois estádios de desenvolvimento da cultura afetaram as características biométricas da cultura assim como a produtividade.
\end{abstract}

Palavras-chave: Biometria da soja. Dossel. Etefon. Lactofem. Produtividade.

\section{USE OF TWO STADIUM GROWTH REGULATORS FOR THE CONTROL OF THE CANOPY IN SOY RR CROP IN SOUTHEAST GOIANO}

\footnotetext{
SUMMARY: Modern cultivars of soybeans with high productive potential are widely tested and diffused in the region, but excessive growth can cause loss of productive potential. One way to solve would be to use growth regulators to control the canopy. The objective of this work was to evaluate the biometry and productivity of soybean cultivar CD 2737 RR throughout the development of the crop after the application of two growth regulators in two stages of growth. The experiment was conducted by two harvests in the agricultural years of 2016/2017 and $2017 / 2018$ in the Experimental Area of the Nucleus of Teaching and Research in Phytotechny, presenting as geographical coordinates, $17^{\circ} 58$ 'S latitude and $45^{\circ} 22^{\prime}$ W longitude and with $845 \mathrm{~m}$ of altitude, in a Quartzarênico Neosol. The experimental design was a randomized complete block design with $4 \times 2 \times 2+1$ with four replications. The treatments were with the herbicide Lactofem $240 \mathrm{gL}-1$, the growth regulator Etefon $720 \mathrm{gL}-1$ in four doses of each and two phenological stages and a control without application. Data were collected from biometric variables, such as: yield in sacks per hectare, weight of a thousand grains, number of pods per plant, number of grains per plant, number of grains per pods, number of nodes per plant, plant height and height insertion of the first pod into the plant.

${ }^{1}$ Pós-Doutor em Fitotecnia (Produção Vegetal) pela Universidade de Coimbra - Portugal. Professor Titular da UniFIMES - Centro Universidade de Mineiros

${ }^{2}$ Acadêmico do curso de Agronomia da UniFimes

${ }^{3}$ Professora Adjunta na UniFimes; Bióloga; Mestre em Botânica

${ }^{4}$ Prof. Adjunto, Doutorando, Fertilidade de Solo, IF-Goiano, campus Rio Verde, diego@unifimes.edu.br

${ }^{5}$ Professor Adjunto na UniFimes; Engenheiro Agrônomo; Doutor em Entomologia pela USP- Universidade de São Paulo

${ }^{6}$ IFMT Campus São Vicente. Departamento de Engenharia Rural e Fitotecnia

${ }^{7}$ Acadêmica do curso de Engenharia Florestal
} 
The data were analyzed by the Sisvar program and submitted to analysis of variance, the means being compared by the $t$ test. It can be concluded that growth regulators used at two stages of crop development affected the biometric characteristics of the crop as well as productivity.

Keyword: Soybean biometry. Canopy. Etefon. Lactofem. Productivity.

\section{INTRODUÇÃO}

Com o aumento dos custos de produção agrícola, influenciado pela cotação dos insumos, quase em sua totalidade importados, e com a instabilidade na política econômica brasileira, uma maneira de se manter a rentabilidade agrícola é melhorando os níveis de produtividade. A soja atualmente tem uma produção anual em nível mundo de 351.311 milhões de toneladas, com uma área plantada de 120.958 milhões de hectares e produção em nível de Brasil de 113.923 milhões de toneladas com uma área plantada de 33.890 milhões de hectares e uma produtividade média de $3.362 \mathrm{kgha}^{-1}$, sendo o estado de Goiás responsável pela produção de 114.03 milhões de toneladas com produtividade média de 3.300 $\mathrm{kg} / \mathrm{ha}^{-1}$ (CONAB, 2017).

Cultivares modernas de soja com elevado potencial produtivo são amplamente testadas e difundidas na região, porém alguns problemas como o crescimento excessivo, o acamamento e o alto sombreamento da cultura, podem atrapalhar seu potencial de rendimento. Isso acontece principalmente em locais com elevada altitude (acima de $700 \mathrm{~m}$ ), em que cultivares de hábito de crescimento indeterminado são utilizadas, em solo com nível de nutrientes elevado e com uma elevada população de plantas. A situação descrita pode ser amenizada ou solucionada com o plantio de outra variedade de soja, reduzindo a adubação ou diminuindo a população de plantas por hectare. Outra alternativa, está no uso de herbicidas ou reguladores de crescimento para reduzir o porte do dossel, o acamamento e o alto sombreamento nessas cultivares (FOLONI, 2016).

O acamamento de plantas provoca perdas significativas no rendimento de grãos da cultura da soja e por isso estratégias de manejo para minimizar o acamamento são de fundamental importância na agricultura atual (BUZZELLO, 2009).

Com o acamamento de plantas é um dos fatores significativos que pode limitar a produção da cultura da soja estar sujeito a intensidade e do estádio de desenvolvimento da cultura em que acontecer, assim como, prejudicar a qualidade dos grãos e a eficiência da colheita mecanizada (SOUZA, et al., 2013)

Portanto, para que seja otimizado o potencial produtivo das cultivares de soja afetada pelo acamamento, o manejo cultural se constitui em uma forma de reduzir ou amenizar o problema. A densidade de plantas, a época de semeadura e a utilização de fitoreguladores são formas de manejo adotadas para evitar o acamamento da soja no campo encontrando um ponto de equilíbrio, o que pode permitir que a cultura expresse seu máximo potencial produtivo (AGROFIT, 2016).

Para alcançar produção de grãos satisfatória que atendam a demanda, é necessário o emprego de práticas de cultivos agrícolas adequados, como o controle do acamamento e também é indispensável à realização de adubação respeitando a recomendação de análise de solo, de modo que a cultura desenvolva em ambiente equilibrado nutricionalmente (ALMEIDA JÚNIOR et al., 2016).

Diante do exposto, este trabalho objetivou avaliar a biometria e a produtividade na cultura da soja, cultivar CD 2737 RR, ao longo do desenvolvimento da cultura, após a aplicação de dois reguladores de crescimento em dois estádios de crescimento. 


\section{MATERIAL E MÉTODO}

O projeto foi conduzido nos anos agrícolas de 2016/2017 e 2017/2108 na área experimental do Núcleo de Estudo e Pesquisa em Fitotecnia, apresentando como coordenadas geográficas aproximadas, $17^{\circ} 58^{\prime} \mathrm{S}$ de latitude e $45^{\circ} 22^{\prime} \mathrm{W}$ de longitude e com $845 \mathrm{~m}$ de altitude. O clima predominante da região, conforme classificação de Köppen (2013) é do tipo Aw, definido como tropical úmido com estação chuvosa no verão e seca no inverno. A precipitação pluvial média anual é de $1.830 \mathrm{~mm}$, com temperatura média anual de aproximadamente $25^{\circ} \mathrm{C}$ e umidade relativa do ar média anual de $66 \%$ conforme a Figura 1 .

O período chuvoso se estende de outubro a março, sendo que os meses de dezembro, janeiro e fevereiro constituem o trimestre mais chuvoso, e o trimestre mais seco corresponde aos meses de junho, julho e agosto (média de $27 \mathrm{~mm}$ ), conforme a Figura 1.

Figura 1 - Temperatura máxima $\left({ }^{\circ} \mathrm{C}\right)$ médias mensais, temperatura média $\left({ }^{\circ} \mathrm{C}\right)$ médias mensais, temperaturas mínimas $\left({ }^{\circ} \mathrm{C}\right)$ médias mensais e precipitação pluvial (mm) acumuladas nas safras2016/2017 e 2017/2018. Mineiros, Goiás. 2018.

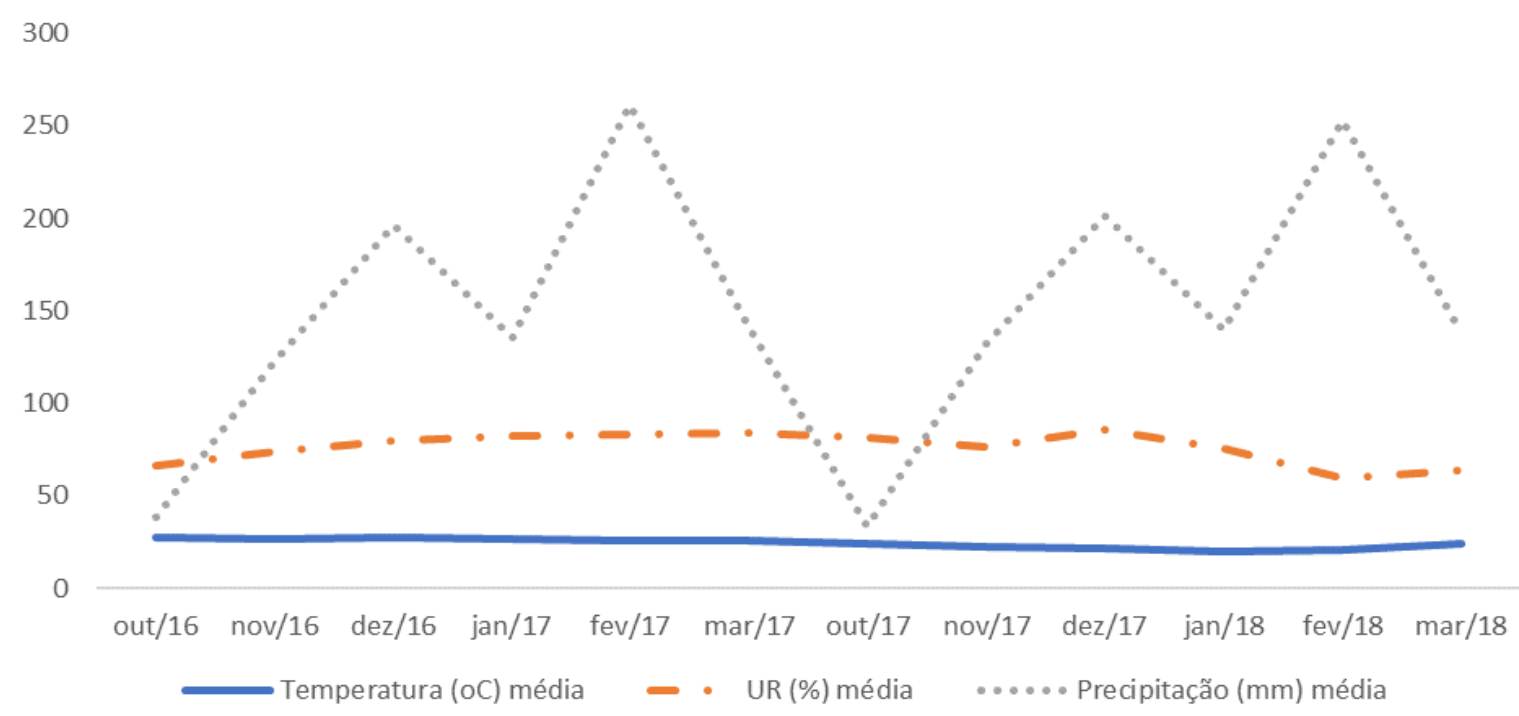

Fonte: AGRITEMPO - Sistema de Monitoramento Agrometeorológico Mineiros / INMET. Mineiros/GO. 2018.

O solo predominante da área, conforme a nova denominação do Sistema Brasileiro de Classificação de Solos Embrapa (2013) é classificado como Neossolo Quartzarênico e de textura arenosa, o qual foi originalmente ocupado por vegetação de Cerrado e vem sendo explorado por culturas anuais há mais de 15 anos.

O delineamento experimental foi em blocos casualizados com esquema $4 \times 2 \times 2+1$ com quatro repetições. Cada parcela experimental foi constituída de quatro linhas de 4,0 metros de comprimento e espaçamento de 0,5 metros ocupou uma área total de $8,0 \mathrm{~m}^{2}(4,0 \mathrm{~m}$ x 0,5 $\mathrm{m}$ x 4,0) e uma área útil de 2,0 $\mathrm{m}^{2}, 2,0$ metros de comprimento e descarte de 1,0 m nas extremidades e 2 linhas centrais com espaçamento entre blocos de 2,0 metros.

Os tratamentos foram constituídos: T1 - Controle (dose zero); T2 - Lactofem $240 \mathrm{~g} \mathrm{~L}^{-1}-0,150 \mathrm{ml}$ p.c.V3; T3 - Lactofem $240 \mathrm{~g} \mathrm{~L}^{-1}$ - 0,300 ml p.c.V3; T4 - Lactofem $240 \mathrm{~g} \mathrm{~L}^{-1}$ - 0,450 ml p.c.V3; T5 Lactofem $240 \mathrm{~g} \mathrm{~L}^{-1}-0,600 \mathrm{ml}$ p.c.V3; T6 - Etefon $720 \mathrm{~g} \mathrm{~L}^{-1}-0,150 \mathrm{ml}$ p.c.V3; T7 - Etefon $720 \mathrm{~g} \mathrm{~L}^{-1}$ 0,300 ml p.c.V3; T8 - Etefon $720 \mathrm{~g} \mathrm{~L}^{-1}$ - 0,450 ml p.c.V3; T9 - Etefon $720 \mathrm{~g} \mathrm{~L}^{-1}$ - 0,600 ml p.c.V3; T10 Lactofem $240 \mathrm{~g} \mathrm{~L}^{-1}$ - 0,150 ml p.c.V11 e V6; T11 - Lactofem $240 \mathrm{~g} \mathrm{~L}^{-1}$ - 0,300 ml p.c.V3 e V6; T12 Lactofem $240 \mathrm{~g} \mathrm{~L}^{-1}$ - 0,450 ml p.c.V3 e V6; T13 - Lactofem $240 \mathrm{~g} \mathrm{~L}^{-1}$ - 0,600 ml p.c.V3 e V6; T14 - 
Etefon $720 \mathrm{~g} \mathrm{~L}^{-1}$ - 0,150 ml p.c.V3 e V6; T15 - Etefon $720 \mathrm{~g} \mathrm{~L}^{-1}$ - 0,300 ml p.c.V3 e V6; T16 - Etefon 720 $\mathrm{g} \mathrm{L}^{-1}-0,450 \mathrm{ml}$ p.c.V3 e V6; T17 - Etefon $720 \mathrm{~g} \mathrm{~L}^{-1}$ - 0,600 ml p.c.V3 e V6.

Os atributos químicos do solo ( $\mathrm{pH}, \mathrm{K}, \mathrm{Ca}, \mathrm{Mg}, \mathrm{H}+\mathrm{Al}$ e $\mathrm{Al}$ ) foram determinados, nas camadas de 0,0 - 0,20 m; 0,20 - 0,40 m segundo a metodologia proposta por Raij e Quaggio (1983), no Laboratório de Fertilidade do Solo da instituição. Esses atributos do solo foram avaliados antes da implantação do projeto de pesquisa de cada safra implantada, para conhecer as características químicas da área experimental conforme a Tabela 1.

Tabela 1- Resultados obtidos na análise química do solo, coletada na área experimental do Núcleo de Ensino e Pesquisa em Fitotecnia, amostrada antes do plantio da soja CD2737 RR. Em função das aplicações de dois reguladores de crescimento em estádios fenológicos diferentes. Mineiros, Goiás. 2018.

\begin{tabular}{|c|c|c|c|c|c|c|c|c|c|c|c|c|}
\hline \multirow{4}{*}{ 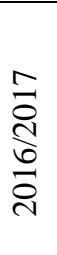 } & Profundidade & $\mathrm{pH}$ & P (Mel) & $\mathrm{K}^{+}$ & $\mathrm{Ca}$ & $\mathrm{Mg}$ & $\mathrm{Al}$ & $\mathrm{H}+\mathrm{Al}$ & S.B. & CTC & $\mathrm{V}$ & M.O. \\
\hline & $(\mathrm{cm})$ & $\mathrm{CaCl}_{2}$ & $\mathrm{mg} \mathrm{dm^{-3 }}$ & \multicolumn{7}{|c|}{ mmolc dm ${ }^{-3}$} & $\%$ & $\mathrm{~g} \mathrm{dm}^{-3}$ \\
\hline & $0-20$ & 4,9 & 7,0 & 1,6 & 18 & 10 & 0 & 31 & 29,8 & 60,8 & 49,05 & 22 \\
\hline & $20-40$ & 4,9 & 6,1 & 1 & 5 & 3 & 0 & 29 & 9 & 38 & 23,76 & 18 \\
\hline & $\begin{array}{l}\text { Profundidade } \\
\text { (cm) }\end{array}$ & $\mathrm{pH}$ & P (Mel) & $\mathrm{K}+$ & $\mathrm{Ca}$ & $\mathrm{Mg}$ & $\mathrm{Al}$ & $\mathrm{H}+\mathrm{Al}$ & S.B. & CTC & $\mathrm{V}$ & M.O. \\
\hline$\infty$ & & $\mathrm{CaCl}_{2}$ & $\mathrm{mg} \mathrm{dm}^{-3}$ & \multicolumn{7}{|c|}{ mmolc dm ${ }^{-3}$} & $\%$ & $\mathrm{~g} \mathrm{dm}^{-3}$ \\
\hline 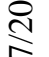 & $0-20$ & 4,5 & 8,0 & 1,4 & 18 & 9 & 0 & 30 & 28,4 & 70,8 & 55,05 & 26 \\
\hline$\overline{\mathrm{\nu}}$ & $20-40$ & 4,4 & 6,8 & 1,2 & 8 & 4 & 0 & 27 & 13,2 & 43 & 27,67 & 21 \\
\hline
\end{tabular}

Fonte: Dados do experimento, 2018.

Na cultivar de soja foram avaliados biometricamente: produtividade em sacas por hectare, peso de mil grãos, número de vagens por planta, número de grãos por planta, número de grãos por vagens, número de nós por planta, altura de planta e altura de inserção da primeira vagem na planta.

Os dados foram analisados pelo programa Sisvar, proposto por Ferreira (2011). Os dados obtidos foram submetidos à análise de variância, sendo as médias comparadas pelo teste de $\mathrm{t}$, quando detectada significância para a ANOVA a $\mathrm{p}=0,05 \%$ de probabilidade para a comparação de médias.

\section{RESULTADOS E DISCUSSÃO}

No resumo da análise de variância (Tabela 2) podemos notar que o bloco safra 2016/2017 ocorreu significância a 5\% de probabilidade na análise de biometria dos parâmetros: produtividade em sacas por hectares, população de planta por hectare, e 1\% de probabilidade na análise biométrica dos parâmetros: altura de planta e altura de inserção da primeira vargem, observando os tratamentos estudados, pode-se notar que os parâmetros da biometria avaliados em que respondeu com significância, foram, produtividade em sacas por hectare, peso de mil grãos, altura de planta e altura de inserção de primeira vagem.

Na safra 2017/2018 registra-se que o bloco ocorreu significância a 1\% de probabilidade na análise de biometria somente no parâmetro peso de mil grãos. Já nos tratamentos foi possível observar significância a $1 \%$ de probabilidade na análise de biometria nos parâmetros: produtividade de sacas por hectare, peso mil grãos, números de nós, número de vagem por plantas e números de grão por plantas. 
Tabela 2 - Resumo de análise de variância (F), estimativa para a biometria da cultura da soja CD2737 RR, conforme tratamentos com aplicação de dois reguladores de crescimento em estádios fenológicos diferentes, safras 2016/2017 e 2017/2018. Mineiros, Goiás. 2018.

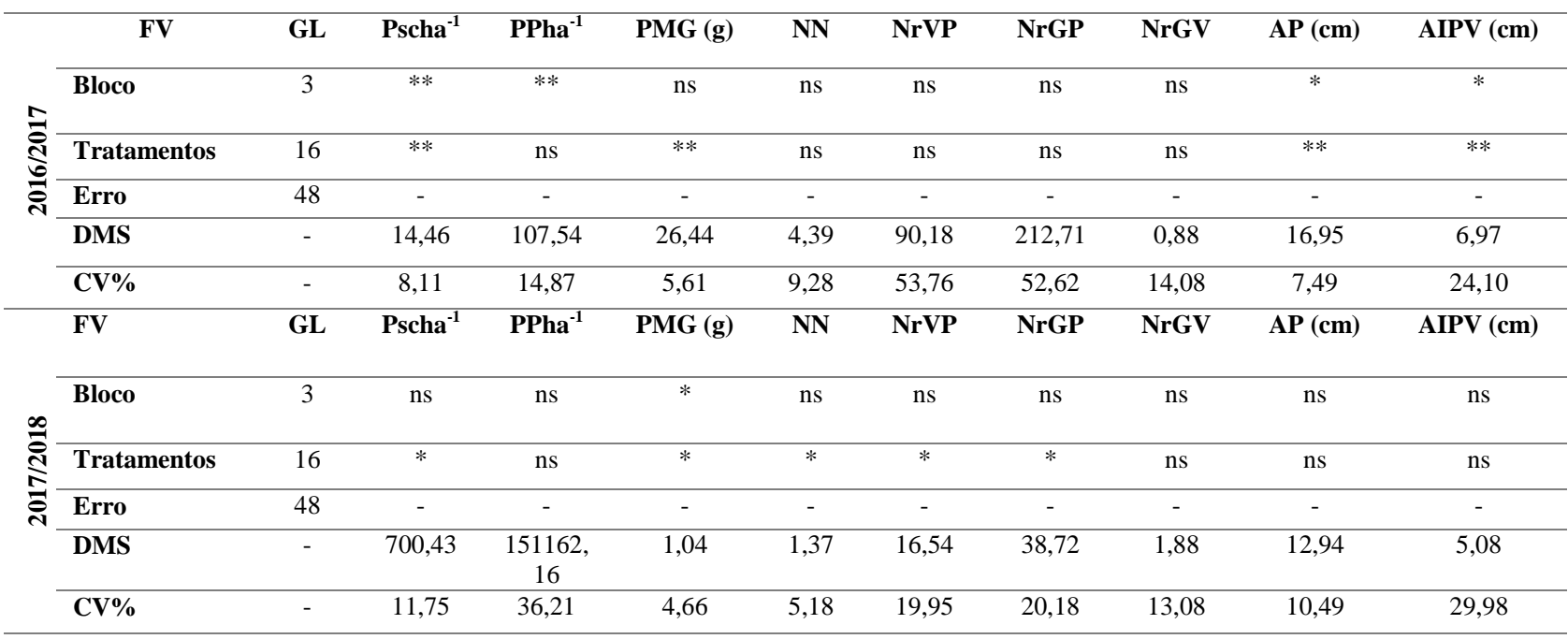

Os símbolos $\left(*^{* *}\right)$ reportam-se a níveis de significância de $1 \%$ e $5 \%$ de probabilidade respectivamente pelo teste F. Pscha $^{-1}$ : Produtividade sacas hectare; PPha $^{-1}$ : População de Plantas; PMG: massa seca de 1000 grão; NN: Número de nós; NrVP: número vagens por planta; NrGP: Número de grãos por planta; NrGV: Número de grãos por vagens; AP: altura de planta; AIPV: altura de inserção de primeira vagem.

Fonte: Dados da pesquisa, 2018.

Permite-se constatar na Tabela 3 as médias de produtividade em sacas por hectare na safra 2016/2017 onde ocorreu diferença estatística significativa, mas ao observar os valores reais em sacas por hectare, nota-se que o tratamento controle, Lactofem $240 \mathrm{~g} \mathrm{~L}^{-1}$ com $0,450 \mathrm{~L}^{-1}$ no V3 e Etefon $720 \mathrm{~g} \mathrm{~L}^{-1}$ com 0,300 $\mathrm{L} \mathrm{ha}^{-1}$ no V3 como seus respectivos valores 76,50; 76,99 e 75,97 $\mathrm{sc} \mathrm{ha}^{-1}$, foram superior aos demais tratamentos, onde os outros tratamentos de uma maneira acentuada ou discreta proporcionaram uma queda na produtividade por hectare com uso dos reguladores de crescimentos aplicados. Para Souza, et al., (2013) com trabalhando realizado à campo e com tratamentos de regulador de crescimento cloreto de clormequate e cloreto de clorocolina as plantas apresentaram diferença significativa entre tratamentos, para variável tecnológica rendimento de grãos. O mesmo resultado foi encontrado neste trabalho.

Nota-se na Tabela 3 que no ano agrícola 2016/2017, não ocorreu diferença significativa na população de planta por hectare. Em trabalho conduzido por Buzzello (2009) também não houve diferença entre os tratamentos quanto ao número de plantas por metro quadrado, afirma ainda que esse fato foi importante para dar maior confiabilidade aos resultados.

Observa-se que no ano agrícola 2017/2018 as médias de produtividade em sacas por hectare apresentaram diferenças significativas, mas ao observar os valores reais em sacas por hectare podemos notar que o tratamento Etefon $720 \mathrm{~g} \mathrm{~L}^{-1}$ com $0,150 \mathrm{~L} \mathrm{ha}^{-1}$ no V3 obteve uma média de 83,78 sc ha ${ }^{-1}$, foi superior aos demais tratamentos na produtividade por hectare (Tabela 3). Em pesquisa à campo realizado por Souza, et al., (2013) com trabalhando realizado à campo e com tratamentos de regulador de crescimento cloreto de clormequate e cloreto de clorocolina as plantas apresentaram diferença significativa entre tratamentos, para variável tecnológica rendimento de grãos. Resultado semelhante foi encontrado nesta pesquisa.

A Tabela 3 registra para o ano agrícola 2017/2018 que a população de planta por hectare apresentou diferença significativa somente para o tratamento Lactofem $240 \mathrm{~g} \mathrm{~L}^{-1}$ com $0,600 \mathrm{~L} \mathrm{ha}^{-1}$ no V3, sendo que todos os outros assemelharam entre si. Resultado semelhante foi encontrado em trabalho conduzido por Buzzello (2009) não encontrando diferença entre os tratamentos quanto ao número 
número de plantas por metro quadrado.

Tabela 3 - Média das variáveis tecnológica, produtividade em sacas por hectare ( $\left.\mathrm{P} \mathrm{Sc} \mathrm{ha}{ }^{-1}\right)$, população de plantas por hectare $\left(\mathrm{PP} \mathrm{ha}^{-1}\right)$. Em função das aplicações de dois reguladores de crescimento em estádios fenológicos em diferentes safras 2016/2017 e 2017/2018. Mineiros, Goiás. 2018.

\begin{tabular}{|c|c|c|c|c|c|c|}
\hline & & & $2016 / 2017$ & $2017 / 2018$ & $2016 / 2017$ & $2017 / 2018$ \\
\hline Tratamentos & Estádio & Dose p. c. (L) & P Sc ha ${ }^{-1}$ & P Sc ha ${ }^{-1}$ & PP ha ${ }^{-1}$ & PP ha ${ }^{-1}$ \\
\hline Controle & - & Zero & $76,50 \mathrm{a}$ & 75,90abcd & 321296 & $315278 \mathrm{ab}$ \\
\hline Lactofem $240 \mathrm{~g} \mathrm{~L}^{-1}$ & V3 & 0,150 & $73,86 \mathrm{ab}$ & $76,23 \mathrm{abcd}$ & 285185 & $319444 \mathrm{ab}$ \\
\hline Lactofem $240 \mathrm{~g} \mathrm{~L}^{-1}$ & V3 & 0,300 & $75,28 \mathrm{ab}$ & $81,18 \mathrm{ab}$ & 292592 & $297222 \mathrm{ab}$ \\
\hline Lactofem $240 \mathrm{~g} \mathrm{~L}^{-1}$ & V3 & 0,450 & 76,99 a & $68,95 \mathrm{cde}$ & 300000 & $268055 \mathrm{ab}$ \\
\hline Lactofem $240 \mathrm{~g} \mathrm{~L}^{-1}$ & V3 & 0,600 & $74,46 \mathrm{ab}$ & 72,69abcde & 287037 & $218055 \mathrm{~b}$ \\
\hline Etefon $720 \mathrm{~g} \mathrm{~L}^{-1}$ & V3 & 0,150 & $67,82 \mathrm{bcd}$ & $83,78 \mathrm{a}$ & 274074 & $255555 \mathrm{ab}$ \\
\hline Etefon $720 \mathrm{~g} \mathrm{~L}^{-1}$ & V3 & 0,300 & $75,97 \mathrm{a}$ & $75,07 \mathrm{abcd}$ & 237037 & $301389 \mathrm{ab}$ \\
\hline Etefon $720 \mathrm{~g} \mathrm{~L}^{-1}$ & V3 & 0,450 & $64,95 \mathrm{~cd}$ & $65,37 \mathrm{de}$ & 285185 & $240277 \mathrm{ab}$ \\
\hline Etefon $720 \mathrm{~g} \mathrm{~L}^{-1}$ & V3 & 0,600 & $67,48 \mathrm{bcd}$ & $75,37 \mathrm{abcd}$ & 278704 & $283333 \mathrm{ab}$ \\
\hline Lactofem $240 \mathrm{~g} \mathrm{~L}^{-1}$ & V3 e V6 & 0,150 & $64,098 \mathrm{~d}$ & $69,35 \mathrm{cde}$ & 265741 & $319444 \mathrm{ab}$ \\
\hline Lactofem $240 \mathrm{~g} \mathrm{~L}^{-1}$ & V3 e V6 & 0,300 & $62,55 \mathrm{~d}$ & $76,41 \mathrm{abcd}$ & 275000 & $372222 \mathrm{a}$ \\
\hline Lactofem $240 \mathrm{~g} \mathrm{~L}^{-1}$ & V3 e V6 & 0,450 & $64,64 \mathrm{~cd}$ & $61,32 \mathrm{e}$ & 276852 & $209722 b$ \\
\hline Lactofem $240 \mathrm{~g} \mathrm{~L}^{-1}$ & V3 e V6 & 0,600 & $65,53 \mathrm{~cd}$ & $63,13 \mathrm{e}$ & 318519 & $277777 \mathrm{ab}$ \\
\hline Etefon $720 \mathrm{~g} \mathrm{~L}^{-1}$ & V3 e V6 & 0,150 & $72,13 \mathrm{abc}$ & $78,61 \mathrm{abcs}$ & 262963 & $290277 \mathrm{ab}$ \\
\hline Etefon $720 \mathrm{~g} \mathrm{~L}^{-1}$ & V3 e V6 & 0,300 & $63,41 \mathrm{~d}$ & $70,18 \mathrm{bcde}$ & 283333 & $293055 \mathrm{ab}$ \\
\hline Etefon $720 \mathrm{~g} \mathrm{~L}^{-1}$ & V3 e V6 & 0,450 & $63,42 \mathrm{~d}$ & $61,81 \mathrm{e}$ & 255556 & $345833 \mathrm{ab}$ \\
\hline Etefon $720 \mathrm{~g} \mathrm{~L}^{-1}$ & V3 e V6 & 0,600 & $65,05 \mathrm{~cd}$ & $74,95 \mathrm{abcd}$ & 262037 & $384722 \mathrm{a}$ \\
\hline DMS & - & - & 7,96 & 700,43 & 107546 & 151162,16 \\
\hline $\mathrm{CV}(\%)$ & - & - & 8,11 & 11,75 & 14,87 & 36,21 \\
\hline
\end{tabular}

Médias seguidas pela mesma letra na coluna não diferem significativamente a $5 \%$ de probabilidade, pelo teste t. p.c.: Produto comercial. Safras 2016/2017 e 2017/2018.

Fonte: Dados da pesquisa, 2018.

Observa-se na Tabela 4 no ano agrícola 2016/2017 na variável tecnológica de peso de mil grãos, ocorreu diferença significativa entre os tratamentos utilizados, o que obteve o melhor peso de mil grãos foi o tratamento controle com uma média de 205 gramas, que se assemelha ao Etefon $720 \mathrm{~g} \mathrm{~L}^{-1}$ com $0,150 \mathrm{~L}$ $\mathrm{ha}^{-1}$ no V3 e V6, Lactofem $240 \mathrm{~g} \mathrm{~L}^{-1}$ com 0,300 L ha- ${ }^{-1}$ no V3 e V6, Lactofem $240 \mathrm{~g} \mathrm{~L}^{-1}$ com 0,450 L ha no V3 e V6, Lactofem $240 \mathrm{~g} \mathrm{~L}^{-1}$ com 0,6000 L ha ${ }^{-1}$ no V3 e V6,Lactofem $240 \mathrm{~g} \mathrm{~L}^{-1}$ com 0,150 L ha ${ }^{-1}$ no V3, Lactofem $240 \mathrm{~g} \mathrm{~L}^{-1}$ com 0,300 $\mathrm{L} \mathrm{ha}^{-1}$ no V3, Lactofem $240 \mathrm{~g} \mathrm{~L}^{-1}$ com 0,450 L ha ${ }^{-1}$ no V3, Lactofem $240 \mathrm{~g} \mathrm{~L}^{-1}$ com $0,600 \mathrm{~L} \mathrm{ha}^{-1}$ no V3, Etefon $720 \mathrm{~g} \mathrm{~L}^{-1}$ com $0,150 \mathrm{~L} \mathrm{ha}^{-1}$ no V3. Souza, et al., (2013) trabalhando com experimento realizado em campo e com tratamentos de regulador de crescimento cloreto de clormequate e cloreto de clorocolina as plantas apresentaram diferença significativa entre tratamentos, para variável tecnológica na massa de mil grãos. O mesmo resultado foi encontrado neste trabalho.

Observa-se na Tabela 4 no ano agrícola 2017/2018 na variável tecnológica peso de mil grãos, onde ocorreu diferença significativa entre os tratamentos utilizados, sendo que o que obteve o melhor peso de mil grãos foram os tratamentos: controle com uma média de 163 gramas que se assemelha com Etefon $720 \mathrm{~g} \mathrm{~L}^{-1}$ com $0,150 \mathrm{~L} \mathrm{ha}^{-1}$ no V3com média de 163 gramas, Etefon $720 \mathrm{~g} \mathrm{~L}^{-1}$ com 0,300 L ha ${ }^{-1}$ no V3 com média de 163 gramas e Etefon $720 \mathrm{~g} \mathrm{~L}^{-1}$ com $0,150 \mathrm{~L} \mathrm{ha}^{-1}$ no V3 e V6 com média de 163 gramas. Nota-se que o resultado com menor gramas por massa de mil grãos foi encontrado no tratamento Etefon $720 \mathrm{~g} \mathrm{~L}^{-1}$ com $0,600 \mathrm{~L} \mathrm{ha}^{-1}$ no V3 e V6 com média de 143 gramas. Em trabalho a campo realizado por Souza, et al., 
(2013) com regulador de crescimento cloreto de clormequate e cloreto de clorocolina as plantas apresentaram diferença significativa entre tratamentos, para variável tecnológica na massa de mil grãos. Resultado semelhante foi encontrado neste trabalho.

Verifica-se na tabela 4 que no resumo de número de vagens por planta não foi possível verificar diferença estatística significativa, sendo que o tratamento controle ficou com 75 vagens por planta e o tratamento que melhor teve rendimento de número de vagens por planta foi Lactofem $240 \mathrm{~g} \mathrm{~L}^{-1}$ com 0,450 $\mathrm{L} \mathrm{ha}^{-1}$ no V3 e V6, com uma média de 116,5 vagens por planta. Observando trabalho realizado por Carvalho, et al. (2013) obteve resultado semelhante a este trabalho, com a variável número de vagens por planta, o tratamento que obteve melhores resultados foram T4 e T5, com 61,67 e 54,625 vagens por planta respectivamente, não diferindo estatisticamente a nível de 5\% com a aplicação de três hormônios vegetais sendo Cinetina, Ácido Giberélico, Ácido4-Indol-3-Ilbutírico.

Verifica-se na Tabela 4, no ano agrícola 2017/2018, para o número de vagens por planta onde foi possível verificar a ocorrência de diferença significativa, sendo que o melhor tratamento foi Lactofem 240 $\mathrm{g} \mathrm{L}^{-1}$ com $0,300 \mathrm{~L} \mathrm{ha}^{-1}$ no V3 que ficou com uma média de 85,75 vagens por planta e o tratamento que obteve o menor rendimento em número de vagens por planta foi o Etefon $720 \mathrm{~g} \mathrm{~L}^{-1}$ com $0,600 \mathrm{Lha}^{-1}$ no V3 ficando com uma média de 34,25 vagens por planta. Resultado contrário foi encontrado por Carvalho, et al., (2013) na variável tecnológica número de vagens por planta, o tratamento que obteve melhores resultados foram T4 e T5, com 61,67 e 54,625 vagens por planta respectivamente, não diferindo estatisticamente a nível de $5 \%$ com a aplicação de três hormônios vegetais sendo Cinetina, Ácido Giberélico, Ácido4-Indol-3-Ilbutírico.

Tabela 4 - Média das variáveis tecnológica, peso de mil grãos (PMG), médias de número de vagens por planta (NrVP). Em função das aplicações de dois reguladores de crescimento em estádios fenológicos diferentes. Safras 2016/2017 e 2017/2018. Mineiros, Goiás. 2018.

(Continua)

\begin{tabular}{|c|c|c|c|c|c|c|}
\hline Tratamentos & Estádio & Dose p. c. (L) & $\begin{array}{l}\text { 2016/2017 } \\
\text { PMG (g) }\end{array}$ & $\begin{array}{l}2017 / 2018 \\
\text { PMG (g) }\end{array}$ & $\begin{array}{l}\text { 2016/2017 } \\
\text { NrVP }\end{array}$ & $\begin{array}{l}2017 / 2018 \\
\text { NrVP }\end{array}$ \\
\hline Controle & - & Zero & $205,0 a$ & $163,0 \mathrm{a}$ & 75,0 & $62,00 \mathrm{~cd}$ \\
\hline Lactofem $240 \mathrm{~g} \mathrm{~L}^{-1}$ & V3 & 0,150 & 190,0 abcde & $160,0 \mathrm{ab}$ & 68,0 & $58,50 \mathrm{cde}$ \\
\hline Lactofem $240 \mathrm{~g} \mathrm{~L}^{-1}$ & V3 & 0,300 & $195,0 \mathrm{abc}$ & $160,0 \mathrm{ab}$ & 60,5 & $85,75 \mathrm{a}$ \\
\hline Lactofem $240 \mathrm{~g} \mathrm{~L}^{-1}$ & V3 & 0,450 & 185,0 abcde & $155,0 \mathrm{abc}$ & 58,0 & $64,25 \mathrm{bc}$ \\
\hline Lactofem $240 \mathrm{~g} \mathrm{~L}^{-1}$ & V3 & 0,600 & 185,0 abcde & $158,0 \mathrm{abc}$ & 53,0 & $63,50 \mathrm{~cd}$ \\
\hline Etefon $720 \mathrm{~g} \mathrm{~L}^{-1}$ & V3 & 0,150 & 182,5 abcde & $163,0 \mathrm{a}$ & 45,8 & $56,25 \mathrm{cdef}$ \\
\hline Etefon $720 \mathrm{~g} \mathrm{~L}^{-1}$ & V3 & 0,300 & 175,0 bcde & $163,0 \mathrm{a}$ & 85,8 & $47,25 \mathrm{defg}$ \\
\hline Etefon $720 \mathrm{~g} \mathrm{~L}^{-1}$ & V3 & 0,450 & 172,5 cde & $160,0 \mathrm{ab}$ & 47,8 & $63,00 \mathrm{~cd}$ \\
\hline Etefon $720 \mathrm{~g} \mathrm{~L}^{-1}$ & V3 & 0,600 & $165,0 \mathrm{e}$ & $160,0 \mathrm{ab}$ & 44,3 & $34,25 \mathrm{~g}$ \\
\hline Lactofem $240 \mathrm{~g} \mathrm{~L}^{-1}$ & V3 e V6 & 0,150 & $165,0 \mathrm{e}$ & $150,0 \mathrm{bcd}$ & 68,5 & $57,25 \mathrm{cdef}$ \\
\hline Lactofem $240 \mathrm{~g} \mathrm{~L}^{-1}$ & V3 e V6 & 0,300 & 187,5 abcde & $150,0 \mathrm{bcd}$ & 62,5 & $68,75 b c$ \\
\hline Lactofem $240 \mathrm{~g} \mathrm{~L}^{-1}$ & V3 e V6 & 0,450 & $192,5 \mathrm{abcd}$ & $160,0 \mathrm{ab}$ & 116,5 & $54,75 \mathrm{cdef}$ \\
\hline Lactofem $240 \mathrm{~g} \mathrm{~L}^{-1}$ & V3 e V6 & 0,600 & 182,5 abcde & $155,0 \mathrm{abc}$ & 91,0 & 47,00defg \\
\hline Etefon $720 \mathrm{~g} \mathrm{~L}^{-1}$ & V3 e V6 & 0,150 & $200,0 \mathrm{ab}$ & $163,0 \mathrm{a}$ & 63,8 & $40,75 f g$ \\
\hline Etefon $720 \mathrm{~g} \mathrm{~L}^{-1}$ & V3 e V6 & 0,300 & 180,0 abcde & $148,0 \mathrm{~cd}$ & 46,0 & $43,25 \mathrm{efg}$ \\
\hline Etefon $720 \mathrm{~g} \mathrm{~L}^{-1}$ & V3 e V6 & 0,450 & $167,5 \mathrm{de}$ & $155,0 \mathrm{abc}$ & 52,5 & $80,50 \mathrm{ab}$ \\
\hline
\end{tabular}


Tabela 4 - Média das variáveis tecnológica, peso de mil grãos (PMG), médias de número de vagens por planta (NrVP). Em função das aplicações de dois reguladores de crescimento em estádios fenológicos diferentes. Safras 2016/2017 e 2017/2018. Mineiros, Goiás. 2018.

(Conclusão)

\begin{tabular}{cccllll}
\hline \multirow{2}{*}{ Tratamentos } & \multirow{2}{*}{ Estádio } & \multirow{2}{*}{ Dose p. c. $(\mathbf{L})$} & $\mathbf{2 0 1 6 / 2 0 1 7}$ & $\mathbf{2 0 1 7 / 2 0 1 8}$ & $\mathbf{2 0 1 6 / 2 0 1 7}$ & $\mathbf{2 0 1 7 / 2 0 1 8}$ \\
\cline { 5 - 7 } & & PMG $(\mathbf{g})$ & PMG $(\mathbf{g})$ & NrVP & NrVP \\
\hline Etefon $720 \mathrm{~g} \mathrm{~L}^{-1}$ & V3 e V6 & 0,600 & $172,5 \mathrm{cde}$ & $143,0 \mathrm{~d}$ & 65,5 & $64,50 \mathrm{bc}$ \\
\hline DMS & - & - & 26,44 & 1,04 & 90,18 & 16,54 \\
\hline CV $(\%)$ & - & - & 5,61 & 4,66 & 53,75 & 19,95
\end{tabular}

Médias seguidas pela mesma letra na coluna não diferem significativamente a 5\% de probabilidade, pelo teste t. p.c.: Produto comercial.

Fonte: Dados da pesquisa, 2018.

Vê-se na Tabela 5 que no ano agrícola 2016/2017 o número de grãos por planta, não foi possível verificar diferença estatística significativa. Resultados semelhante foram obtidos por Carvalho et al. (2013), para a variável número de grãos por vagem, não houve diferença entre os tratamentos testados composto por três hormônios vegetais sendo Cinetina, Ácido Giberélico, Ácido4-Indol-3-Ilbutírico.

Vê-se na Tabela 5 em que no ano agrícola 2017/2018 o número de grãos por planta ocorreu diferença significativa entre os tratamentos utilizados, onde o tratamento Lactofem $240 \mathrm{~g} \mathrm{~L}^{-1}$ com $0,300 \mathrm{~L}$ ha $^{-1}$ no V3 foi o que obteve a melhor média com 192 grãos por planta. Em quanto, que, o tratamento Etefon $720 \mathrm{~g} \mathrm{~L}^{-1}$ com $0,600 \mathrm{~L} \mathrm{ha}^{-1}$ no V3 obteve a menor média, ficando com 83,50 grãos por planta. Resultado contrário foi encontrado por Linzmeyer Junior et al. (2008), pois a aplicação de trinexapacethyl na cultura de soja, cultivar CD 209, nas doses de 50, 100, 150, 200 e $250 \mathrm{~g}_{\text {ha- }}{ }^{1}$ não influenciou o número de grãos por planta.

Visualiza-se na Tabela 5 que no ano agrícola 2017/2018 a variável tecnológica número de grãos por vagens não obteve diferença significativa entre os tratamentos. Resultado semelhante foi encontrado por Ávila et al. (2008) indicam que a aplicação de Stimulate ${ }^{\circledR}$ (AIB+GA3+cinetina) no maior nível $(0,00375+0,00375+0,00675$ ha-1), aplicado via foliar, não influenciou o número de grãos por vagens de soja na cultivar CD 202.

Tabela 5 - Médias das variáveis tecnológica, número de grãos por planta (NrGP), número de grãos por vagens $(\mathrm{NrGV})$.Em função das aplicações de dois reguladores de crescimento em estádios fenológicos diferentes. Mineiros, Goiás. 2018.

(Continua)

\begin{tabular}{|c|c|c|c|c|c|c|}
\hline & & & $2016 / 2017$ & $2017 / 2018$ & $2016 / 2017$ & $2017 / 2018$ \\
\hline Tratamentos & Estádio & Dose p. c. (L) & NrGP & NrGP & NrGV & NrGV \\
\hline Controle & - & Zero & 178,75 & $141 \mathrm{cde}$ & 2,32 & 2,33 \\
\hline Lactofem $240 \mathrm{~g} \mathrm{~L}^{-1}$ & $\mathrm{~V} 3$ & 0,150 & 159,50 & $139 \mathrm{cde}$ & 2,33 & 2,43 \\
\hline Lactofem $240 \mathrm{~g} \mathrm{~L}^{-1}$ & V3 & 0,300 & 137,75 & $192 \mathrm{a}$ & 2,34 & 2,36 \\
\hline Lactofem $240 \mathrm{~g} \mathrm{~L}^{-1}$ & V3 & 0,450 & 132,50 & $155 \mathrm{abc}$ & 2,30 & 2,31 \\
\hline Lactofem $240 \mathrm{~g} \mathrm{~L}^{-1}$ & $\mathrm{~V} 3$ & 0,600 & 129,00 & $145 \mathrm{~cd}$ & 2,44 & 2,43 \\
\hline Etefon $720 \mathrm{~g} \mathrm{~L}^{-1}$ & $\mathrm{~V} 3$ & 0,150 & 114,50 & $128,3 \mathrm{cdefg}$ & 2,50 & 2,51 \\
\hline Etefon $720 \mathrm{~g} \mathrm{~L}^{-1}$ & $\mathrm{~V} 3$ & 0,300 & 204,75 & 111,0defgh & 2,43 & 2,45 \\
\hline Etefon $720 \mathrm{~g} \mathrm{~L}^{-1}$ & V3 & 0,450 & 129,25 & $155,3 \mathrm{abc}$ & 2,64 & 2,63 \\
\hline Etefon $720 \mathrm{~g} \mathrm{~L}^{-1}$ & $\mathrm{~V} 3$ & 0,600 & 107,50 & $83,50 \mathrm{~h}$ & 2,43 & 2,44 \\
\hline
\end{tabular}


Tabela 5 - Médias das variáveis tecnológica, número de grãos por planta (NrGP), número de grãos por vagens $(\mathrm{NrGV})$.Em função das aplicações de dois reguladores de crescimento em estádios fenológicos diferentes. Mineiros, Goiás. 2018.

(Conclusão)

\begin{tabular}{|c|c|c|c|c|c|c|}
\hline & & & $2016 / 2017$ & $2017 / 2018$ & $2016 / 2017$ & $2017 / 2018$ \\
\hline Tratamentos & Estádio & Dose p. c. (L) & NrGP & NrGP & NrGV & NrGV \\
\hline Lactofem $240 \mathrm{~g} \mathrm{~L}^{-1}$ & V3 e V6 & 0,150 & 194,75 & $131,0 \mathrm{cdef}$ & 2,99 & 3,00 \\
\hline Lactofem $240 \mathrm{~g} \mathrm{~L}^{-1}$ & V3 e V6 & 0,300 & 150,75 & $158,3 \mathrm{abc}$ & 2,48 & 2,50 \\
\hline Lactofem $240 \mathrm{~g} \mathrm{~L}^{-1}$ & V3 e V6 & 0,450 & 266,75 & $126,5 \mathrm{cdefg}$ & 2,29 & 2,30 \\
\hline Lactofem $240 \mathrm{~g} \mathrm{~L}^{-1}$ & V3 e V6 & 0,600 & 213,25 & $104,5 \mathrm{efgh}$ & 2,38 & 2,36 \\
\hline Etefon $720 \mathrm{~g} \mathrm{~L}^{-1}$ & V3 e V6 & 0,150 & 155,00 & $90,50 \mathrm{gh}$ & 2,47 & 2,45 \\
\hline Etefon $720 \mathrm{~g} \mathrm{~L}^{-1}$ & V3 e V6 & 0,300 & 115,50 & $97,0 \mathrm{fgh}$ & 2,49 & 2,47 \\
\hline Etefon $720 \mathrm{~g} \mathrm{~L}^{-1}$ & V3 e V6 & 0,450 & 128,50 & $184,5 \mathrm{ab}$ & 2,44 & 2,45 \\
\hline Etefon $720 \mathrm{~g} \mathrm{~L}^{-1}$ & V3 e V6 & 0,600 & 142,75 & $152,50 \mathrm{bc}$ & 2,17 & 2,19 \\
\hline DMS & 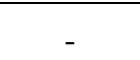 & 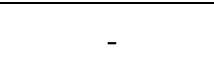 & 212,71 & 38,72 & 0,89 & 1,88 \\
\hline CV $(\%)$ & - & - & 52,62 & 20,18 & 14,08 & 13,08 \\
\hline
\end{tabular}

Médias seguidas pela mesma letra na coluna não diferem significativamente a $5 \%$ de probabilidade, pelo teste t. p.c.: Produto comercial.

Fonte: Dados da pesquisa, 2018.

Detecta-se na Tabela 6 no ano agrícola 2016/2017 onde as médias de número de nós por planta não ocorreu diferença significativa para esta variável da biometria avaliada, mas nota-se que o tratamento controle o número de nós foi 18,25 em média e o melhor tratamento, ficou com uma média de 20,25 com o tratamento de Lactofem $240 \mathrm{~g} \mathrm{~L}^{-1}$ com $0,150 \mathrm{~L} \mathrm{ha}^{-1}$ no V3 e V6, onde podemos perceber um acréscimo no número de nós por planta.

Registra-se na Tabela 6, no ano agrícola 2016/2017 que a média para variável tecnológica altura de planta, demonstrou diferença significativa entre os tratamentos testados, onde o tratamento Lactofem $240 \mathrm{~g} \mathrm{~L}^{-1}$ com $0,450 \mathrm{~L} \mathrm{ha}^{-1}$ no V3 foi o resultado com menor eficiência no controle do crescimento e o tratamento Lactofem $240 \mathrm{~g} \mathrm{~L}^{-1}$ com $0,600 \mathrm{~L} \mathrm{ha}^{-1}$ no V3 e V6 foi o resultado com melhor índice de eficiência, isto é, causando uma perda real na altura de planta. Souza et al., (2013) trabalhando com o cloreto de clormequate e cloreto de clorocolina, as plantas apresentaram as menores estaturas, quando comparados à testemunha (sem aplicação de redutor) promovendo diferenças estatísticas significativas entre os tratamentos testados. Resultados semelhante foi encontrado neste trabalho.

Registra-se na Tabela 6 no ano agrícola 2017/2018 onde as médias de número de nós por planta ocorreram diferença significativa nesta variável biométrica, mas pode-se notar que o tratamento que menos reduziu o número de nós, ficando com uma média de 20,00 nós, foi o tratamento de Etefon $720 \mathrm{~g}$ $\mathrm{L}^{-1}$ com $0,600 \mathrm{~L} \mathrm{ha}^{-1}$ no V3e V6, em que se pode perceber um acréscimo no número de nós por planta, isto é, maior altura de planta. Nota-se também que os tratamentos com menores números de nós foram Lactofem $240 \mathrm{~g} \mathrm{~L}^{-1}$ com 0,600 $\mathrm{La}^{-1}$ no V3 e V6 e Etefon $720 \mathrm{~g} \mathrm{~L}^{-1}$ com 0,150 $\mathrm{L} \mathrm{ha}^{-1}$ no V3 e V6 com uma média de nós de 17,25 e 17,50 respectivamente, foi o que melhor teve controle de altura nas plantas estudada. Em trabalho realizado por Souza, et al., (2013) foi encontrado resultados semelhantes quando testou

Registra-se na Tabela 6, no ano agrícola 2017/2018 a média para altura de planta que ocorreu diferença significativa entre os tratamentos testados, onde o tratamento controle com zero $\mathrm{L} \mathrm{ha}^{-1}$ foi que se destacou como o pior resultado, chegando a uma média de $100,75 \mathrm{~cm}$, sem nenhum controle de altura nas plantas testadas. No entanto, o tratamento Lactofem $240 \mathrm{~g} \mathrm{~L}^{-1}$ com $0,600 \mathrm{~L} \mathrm{ha}^{-1}$ no V3 e V6 foi o resultado 
com menor altura de planta, com uma média de $76,50 \mathrm{~cm}$ causando uma perda real na altura de planta. Resultados semelhante foi encontrado por Rodrigues et al., (2015) onde o comprimento de parte aérea sofreu a influência positiva das doses crescentes de Stimulate ${ }^{\circledR}(0,250,500,750$ e $1000 \mathrm{ml}$ para $100 \mathrm{~kg}$ de sementes), utilizando a cultivar de arroz BRS Primavera. Resultado semelhante foi encontrado em trabalho realizado por (SOUZA et al., 2013), em que a estatura das plantas testadas promovera diferenças estatísticas, sendo que os tratamentos com o cloreto de clormequate e cloreto de clorocolina as plantas apresentaram as menores estaturas, quando comparados à testemunha (sem aplicação de redutor), resultados semelhante foi encontrado neste trabalho.

Tabela 6 - Médias das variáveis tecnológicas, número de nós por planta (NN), altura de planta (AP). Em função das aplicações de dois reguladores de crescimento em estádios fenológicos diferentes. Mineiros, Goiás. 2018.

\begin{tabular}{|c|c|c|c|c|c|c|}
\hline & & & $2016 / 2017$ & $2017 / 2018$ & $2016 / 2017$ & $2017 / 2018$ \\
\hline Tratamentos & Estádio & Dose p. c. (L) & NN & NN & $\mathbf{A P}(\mathbf{c m})$ & $\mathbf{A P}(\mathbf{c m})$ \\
\hline Controle & - & Zero & 18,25 & $19,25 \mathrm{cdef}$ & $92.75 \mathrm{~cd}$ & $100,75 d$ \\
\hline Lactofem $240 \mathrm{~g} \mathrm{~L}^{-1}$ & V3 & 0,150 & 17,75 & $19,75 \mathrm{ef}$ & $93,25 \mathrm{~cd}$ & $94,00 \mathrm{~cd}$ \\
\hline Lactofem $240 \mathrm{~g} \mathrm{~L}^{-1}$ & V3 & 0,300 & 19,00 & $19,00 \mathrm{bcdef}$ & $90,00 \mathrm{bcd}$ & $88,75 \mathrm{abcd}$ \\
\hline Lactofem $240 \mathrm{~g} \mathrm{~L}^{-1}$ & V3 & 0,450 & 18,00 & $19,50 \mathrm{bcd}$ & $96,75 \mathrm{c}$ & $83,00 \mathrm{abc}$ \\
\hline Lactofem $240 \mathrm{~g} \mathrm{~L}^{-1}$ & V3 & 0,600 & 17,75 & $18,00 \mathrm{abc}$ & $88,75 \mathrm{bcd}$ & $81,50 \mathrm{abc}$ \\
\hline Etefon $720 \mathrm{~g} \mathrm{~L}^{-1}$ & V3 & 0,150 & 17,00 & $19,50 \mathrm{def}$ & $93,25 \mathrm{~cd}$ & $91,50 \mathrm{~cd}$ \\
\hline Etefon $720 \mathrm{~g} \mathrm{~L}^{-1}$ & V3 & 0,300 & 18,25 & 18,50abcde & $92,00 \mathrm{bcd}$ & $86,00 \mathrm{abc}$ \\
\hline Etefon $720 \mathrm{~g} \mathrm{~L}^{-1}$ & V3 & 0,450 & 17,75 & $17,75 \mathrm{ab}$ & $88,25 \mathrm{bcd}$ & $86,25 \mathrm{abc}$ \\
\hline Etefon $720 \mathrm{~g} \mathrm{~L}^{-1}$ & V3 & 0,600 & 17,75 & $18,00 \mathrm{abc}$ & $91,25 \mathrm{bcd}$ & $90,00 \mathrm{~cd}$ \\
\hline Lactofem $240 \mathrm{~g} \mathrm{~L}^{-1}$ & V3 e V6 & 0,150 & 20,25 & 18,50abcde & $87,00 \mathrm{bcd}$ & $83,00 \mathrm{abc}$ \\
\hline Lactofem $240 \mathrm{~g} \mathrm{~L}^{-1}$ & V3 e V6 & 0,300 & 17,00 & $19,75 \mathrm{ef}$ & $78,00 \mathrm{bc}$ & $83,00 \mathrm{abc}$ \\
\hline Lactofem $240 \mathrm{~g} \mathrm{~L}^{-1}$ & V3 e V6 & 0,450 & 18,00 & $18,25 \mathrm{abcd}$ & $76,75 b c$ & $81,75 \mathrm{abc}$ \\
\hline Lactofem $240 \mathrm{~g} \mathrm{~L}^{-1}$ & V3 e V6 & 0,600 & 18,75 & $17,25 \mathrm{a}$ & $75,50 \mathrm{a}$ & $76,50 \mathrm{a}$ \\
\hline Etefon $720 \mathrm{~g} \mathrm{~L}^{-1}$ & V3 e V6 & 0,150 & 19,50 & $17,50 \mathrm{a}$ & $93,50 \mathrm{~cd}$ & $92,25 \mathrm{~cd}$ \\
\hline Etefon $720 \mathrm{~g} \mathrm{~L}^{-1}$ & V3 e V6 & 0,300 & 18,75 & $18,25 \mathrm{abcd}$ & $88,50 \mathrm{bcd}$ & $87,75 \mathrm{abc}$ \\
\hline Etefon $720 \mathrm{~g} \mathrm{~L}^{-1}$ & V3 e V6 & 0,450 & 19,00 & $18,25 \mathrm{abcd}$ & $86,75 \mathrm{bcd}$ & $78,50 \mathrm{ab}$ \\
\hline Etefon $720 \mathrm{~g} \mathrm{~L}^{-1}$ & $\mathrm{~V} 3$ e V6 & 0,600 & 18,75 & $20 \mathrm{f}$ & $77,25 \mathrm{bc}$ & $88,50 \mathrm{abcd}$ \\
\hline DMS & - & - & 4,39 & 1,37 & 16,94 & 12,94 \\
\hline $\mathrm{CV}(\%)$ & - & - & 9,28 & 5,18 & 7,49 & 10,49 \\
\hline
\end{tabular}

Médias seguidas pela mesma letra na coluna não diferem significativamente a 5\% de probabilidade, pelo teste t. p.c.: Produto comercial.

Fonte: Dados da pesquisa, 2018.

Na tabela 7 estão registradas as médias do ano agrícola 2016/2017, da variável tecnológica altura de inserção de primeira vagem que obteve diferença significativa entre os tratamentos testados, no qual o tratamento Etefon $240 \mathrm{~g} \mathrm{~L}^{-1}$ com 0,300 L ha- ${ }^{-1}$ no V3 e V6 que foi o tratamento que obteve o pior resultado, com uma altura média de inserção de primeira vagem de 17,00 centímetros e o resultado com maior eficiência do produto utilizado foi o Lactofem $240 \mathrm{~g} \mathrm{~L}^{-1}$ com $0,300 \mathrm{~L} \mathrm{ha}^{-1}$ no V3, com uma média de altura de inserção de primeira vagem de 9,00 centímetros. Resultado semelhante foi encontrado por Bertolin et al. (2010) os resultados médios para altura de plantas, ramos por planta e altura de inserção da primeira 
vagem foram de 83, 14 e 14,4 cm respectivamente, informa ainda que estes valores favorecem a colheita mecânica das plantas pois, cultivares com altura de planta igual ou superior a $65 \mathrm{~cm}$ e ponto de inserção das primeiras vagens igual ou superior a $10 \mathrm{~cm}$ são desejáveis para a realização da colheita mecânica, como se observa, os dados obtidos neste trabalho foram semelhante aos valores mínimos indicados pela literatura.

Na tabela 7, no ano agrícola 2017/2018 as médias para variável tecnológica altura de inserção de primeira vagem obteve diferença significativa entre os tratamentos utilizados, no qual o tratamento Lactofem $240 \mathrm{~g} \mathrm{~L}^{-1}$ com $0,600 \mathrm{~L} \mathrm{ha}^{-1}$ no V3 e V6 com uma média de 9,50 centímetros foi o tratamento com melhor eficiência em comparação como outros resultado testados e o tratamento com menor resultados foi o Lactofem $240 \mathrm{~g} \mathrm{~L}^{-1}$ com 0,600L ha ${ }^{-1}$ no V3 com uma média de 15,25.Resultados semelhante foi encontrado por Bertolin et al. (2010) os resultados médios para altura de plantas, ramos por planta e altura de inserção da primeira vagem foram de 8,3,14 e 14,4 cm respectivamente, informa ainda que estes valores favorecem a colheita mecânica das plantas pois, cultivares com altura de planta igual ou superior a $65 \mathrm{~cm}$ e ponto de inserção das primeiras vagens igual ou superior a $10 \mathrm{~cm}$ são desejáveis para a realização da colheita mecânica.

Tabela 7 - Médias das variáveis tecnológicas, altura da inserção da primeira vagem (AIPV). Em função das aplicações de dois reguladores de crescimento em estádios fenológicos diferentes. Mineiros, Goiás. 2018.

\begin{tabular}{|c|c|c|c|c|}
\hline & & & $2016 / 2017$ & $2017 / 2018$ \\
\hline Tratamentos & Estádio & Dose p. c. (L) & AIPV (cm) & AIPV (cm) \\
\hline Controle & - & Zero & $9,50 \mathrm{~cd}$ & $10,50 \mathrm{abc}$ \\
\hline Lactofem $240 \mathrm{~g} \mathrm{~L}^{-1}$ & V3 & 0,150 & $9,50 \mathrm{~cd}$ & $13,00 \mathrm{abc}$ \\
\hline Lactofem $240 \mathrm{~g} \mathrm{~L}^{-1}$ & V3 & 0,300 & $9,00 \mathrm{a}$ & $10,00 \mathrm{bc}$ \\
\hline Lactofem $240 \mathrm{~g} \mathrm{~L}^{-1}$ & V3 & 0,450 & $9,50 \mathrm{~cd}$ & $12,75 \mathrm{abc}$ \\
\hline Lactofem $240 \mathrm{~g} \mathrm{~L}^{-1}$ & V3 & 0,600 & $9,75 \mathrm{bcd}$ & $15,25 \mathrm{c}$ \\
\hline Etefon $720 \mathrm{~g} \mathrm{~L}^{-1}$ & $\mathrm{~V} 3$ & 0,150 & $10,00 \mathrm{bcd}$ & $13,50 \mathrm{abc}$ \\
\hline Etefon $720 \mathrm{~g} \mathrm{~L}^{-1}$ & V3 & 0,300 & $10,50 \mathrm{abcd}$ & $10,50 \mathrm{abc}$ \\
\hline Etefon $720 \mathrm{~g} \mathrm{~L}^{-1}$ & V3 & 0,450 & $12,25 \mathrm{abcd}$ & $13,00 \mathrm{abc}$ \\
\hline Etefon $720 \mathrm{~g} \mathrm{~L}^{-1}$ & V3 & 0,600 & $9,50 \mathrm{~cd}$ & $11,75 \mathrm{abc}$ \\
\hline Lactofem $240 \mathrm{~g} \mathrm{~L}^{-1}$ & V3 e V6 & 0,150 & $10,50 \mathrm{abcd}$ & $10,75 \mathrm{abc}$ \\
\hline Lactofem $240 \mathrm{~g} \mathrm{~L}^{-1}$ & V3 e V6 & 0,300 & $9,50 \mathrm{~cd}$ & $11,50 \mathrm{abc}$ \\
\hline Lactofem $240 \mathrm{~g} \mathrm{~L}^{-1}$ & V3 e V6 & 0,450 & $10,00 \mathrm{bcd}$ & $10,75 \mathrm{abc}$ \\
\hline Lactofem $240 \mathrm{~g} \mathrm{~L}^{-1}$ & V3 e V6 & 0,600 & $9,50 \mathrm{~cd}$ & $9,50 \mathrm{a}$ \\
\hline Etefon $720 \mathrm{~g} \mathrm{~L}^{-1}$ & V3 e V6 & 0,150 & $16,50 \mathrm{ab}$ & $12,75 \mathrm{abc}$ \\
\hline Etefon $720 \mathrm{~g} \mathrm{~L}^{-1}$ & V3 e V6 & 0,300 & $17,00 \mathrm{~d}$ & $10,75 \mathrm{abc}$ \\
\hline Etefon $720 \mathrm{~g} \mathrm{~L}^{-1}$ & V3 e V6 & 0,450 & $16,25 a b c$ & $11,75 \mathrm{abc}$ \\
\hline Etefon $720 \mathrm{~g} \mathrm{~L}^{-1}$ & $\mathrm{~V} 3 \mathrm{e} \mathrm{V} 6$ & 0,600 & $11,50 \mathrm{abcd}$ & $14,75 \mathrm{bc}$ \\
\hline DMS & - & - & & 5,08 \\
\hline CV (\%) & - & - & & 29,98 \\
\hline
\end{tabular}

Médias seguidas pela mesma letra na coluna não diferem significativamente a 5\% de probabilidade, pelo teste t. p.c.: Produto comercial.

Fonte: Dados da pesquisa, 2018. 


\section{CONCLUSÃO}

Pode-se concluir que os reguladores de crescimentos utilizados em dois estádios de desenvolvimento da cultura afetaram as características biométricas da cultura e a produtividade, podendo acarretar perdas na produtividade na cultura da soja.

\section{AGRADECIMENTOS}

Ao Núcleo de Ensino e Pesquisa em Fitotecnia por contribuir com informações técnicas e cientifica no desenvolvimento destes projetos. A todos os acadêmicos do curso de Engenharia Agronômica pela participação no desenvolvimento deste projeto.

\section{REFERÊNCIAS}

AGROFIT C. A. Ministério da Agricultura, Pecuária e Abastecimento. Coordenação Geral de Agrotóxicos e Afins. DFIA/DAS. 2016.

AGRITEMPO - Sistema de Monitoramento Agrometeorológico Mineiros. Estação TRMM.2334. Mineiros. Goiás. 2018. https://www.agritempo.gov.br/agritempo/jsp/Estacao/index.jsp?siglaUF=GO

ALMEIDA JÚNIOR, J. J. et al. Utilização de Adubação Organomineral na Cultura da Soja. II Colóquio Estadual e Pesquisa Multidisciplinar, 2016.

ÁVILA, M. R; BLANK, A. F; REZENDE, P. M. Aplicação de fito regulador, desempenho agronômico e qualidade de sementes de soja. Scientia agrícola. (Piracicaba, Braz.), v.65, n.6, p.604-612. 2008.

BERTOLIN, D. C. et al. Aumento da produtividade de soja com a aplicação de bioestimulantes. Bragantia, Campinas, v.69, n.2, p.339-347, 2010.

BUZZELLO, G. L. Uso de reguladores no controle do crescimento e no desempenho agronômico da cultura da soja cultivar, CD 214 RR. Dissertação (Mestrado) - Universidade Tecnológica Federal do Paraná. Programa de Pós-Graduação em Agronomia. Pato Branco, 2009.

CARVALHO, C. G. P.et al.. Proposta de classificação dos coeficientes de variação em relação á produtividade e altura da planta de soja. Pesquisa agropecuária brasileira. Brasília-DF. V.38, n.2, p. 187-193, fevereiro, 2003. ISSN 1678-3921

CARVALHO, E. R.et al. Fertilizante mineral e resíduo orgânico sobre características agronômicas da soja e nutrientes no solo. Revista Ciência Agronômica, v. 42, n. 4, p. 930-939, out-dez, 2011.http://dx.doi.org/10.1590/S1806-66902011000400015.

CARVALHO, J. C; VIECELLI, C. A; DE ALMEIDA, D. K. Produtividade e desenvolvimento da cultura da soja pelo uso de regulador vegetal. Acta Iguazu, Cascavel, v.2, n.1, p. 50-60, 2013. ISSN: 2316-4093

CONAB - Companhia Nacional de Abastecimento. Acompanhamento da Safra Brasileira: Grãos. 10. ed. Brasília: Observatório Agrícola, 2017. 119 p.

EMBRAPA - Empresa Brasileira de Pesquisa Agropecuária. Sistema Brasileiro de Classificação de Solos. 3.ed. Brasília, 2013. 353 p. 
FERREIRA, D. F; SISVAR: A Guide for its Bootstrap procedure in multiplecomparisons. Ciência e Agrotecnologia. [online]. 2014, vol.38, n.2, pp. 109-112. 2011. Disponível em: ISSN 1413-7054. http://dx.doi.org/10.1590/S1413-0542014000200001.

FOLONI, J.S.S.de et al. Lactofem e Etefom como reguladores de crescimento de cultivares de soja. In: REUNIÃO DE PESQUISA DE SOJA, 35, jul. 2016. Londrina/PR. Resumos expandidos [...]

KÖPPEN, G. et al.Köppen's Climate Classification Map for Brazil. (em inglês). Meteorologische Zeitschrift , 2013. 711-728.

LINZMEYER JUNIOR, R.et al. Influência de regulador vegetal e densidades de plantas sobre o crescimento, acamamento e produtividade da soja. Acta Scientiarum Agronomy, Maringá, v.30, n.3, p.373-379, 2008.

NAKAYAMA, F. T.; PINHEIRO, G. A. S.; ZERBINI, E. F. Eficiência do fertilizante organomineral na produtividade do feijoeiro (Phaseolusvulgaris L.) em sistema de semeadura direta. IX Fórum Ambiental da Alta Paulista. Periódico Eletrônico v.9, n.7, p. 122-138, 2013. ISSN 1980-0827. DOI: $10.17271 / 19800827$.

RAIJ, B. V.; QUAGGIO, J. A. Métodos de Análise de Solo para Fins de Fertilidade. Campinas, Instituto Agronômico, 1983. 31p. (Boletim técnico, 81).

RODRIGUES, L. Aet al. Avaliação Fisiológica de Sementes de Arroz Submetidas a Doses de Bioestimulante. Nucleus, Ituverava, v. 12, n. 1, p. 207-214, apr. 2015. ISSN 1982-2278. Disponível em: 〈http://www.nucleus.feituverava.com.br/index.php/nucleus/article/view/1376/1762>. Acesso em: 18 jul. 2018. doi:http://dx.doi.org/10.3738/1982.2278.1376.

SOUZA, C. A.et al.Arquitetura de Plantas e Produtividade da Soja Decorrente do uso de Redutores de Crescimento. Bioscience Journal. Uberlandia, v. 29, n. 3, p. 634-643, May/June 2013 http://www.seer.ufu.br/index.php/biosciencejournal/issue/view/973. ISSN 1981-3163 - Online Journal. 